\title{
Model-based and data-driven model-reference control: a comparative analysis
}

\author{
Simone Formentin, Klaske van Heusden and Alireza Karimi
}

\begin{abstract}
In many industrial applications, finding a model from physical laws that is both simple and reliable for control design is a hard and time-consuming undertaking. When a set of input/output (I/O) measurements is available, one can derive the controller directly from data, without relying on the knowledge of the physics. In the scientific literature, two main approaches have been proposed for control system design from data. In the "model-based" approach, a model of the system is first derived from data and then a controller is computed based on the model. In the "data-driven" approach, the controller is directly computed from data. In this work, the above approaches are compared from a novel perspective. The main finding of the paper is that, although from the standard perspective of parameter variance analysis the model-based approach is always statistically more efficient, the data-driven controller might outperform the model-based solution for what concerns the final control cost.
\end{abstract}

\section{INTRODUCTION}

In the last decade, the progress of data-acquisition technology has made it easy and straightforward to collect a large amount of measurements from industrial plants. The use of data as an alternative to physical knowledge to design fixed-order controllers, e.g. PID, has attracted more and more interest throughout the years, since it is often cheaper and less time-consuming. Specifically, two main approaches have been studied in the scientific literature.

In the "model-based" approach, a model of the plant is identified from data and used to compute the fixed-order controller satisfying some user-defined requirements. As an example, in model reference control, the identified model is used to design a controller that minimizes the model reference criterion, either algebraically or through optimization, and a controller-order reduction step is performed (if needed) before implementation. However, this controller is not necessarily optimal when connected to the plant, and the control performance is limited by modeling errors.

In the "data-driven" controller tuning approach, the controller is directly derived from input/output (I/O) data. These techniques have been proposed to avoid the problem of under-modeling and to facilitate the design of fixed-order controllers, both iteratively [7], [15], [11], [8] and noniteratively [5], [1], [21]. Specifically, in non-iterative approaches, stability can be guaranteed [21] and, since the controller parameter estimation problem is convex for most

Laboratoire d'Automatique, École Polytechnique Fédérale de Lausanne (EPFL), 1015 Lausanne, Switzerland.

Simone Formentin is currently a post-doctoral fellow at the University of Bergamo, Italy. Klaske van Heusden is currently a post-doctoral fellow at the University of British Columbia, Canada.

Corresponding author: alireza.karimi@epfl.ch interesting controller structures, the global optimum can be found. Various application examples (e.g., [4], [3]) have shown that critical control problems can be dealt with by using a data-driven method. However, it can be debated whether similar results can be obtained if the same amount of data is available for system identification and a modelbased controller tuning approach is used.

In the context of system identification, it has been shown that an indirect approach consisting of two optimization steps is statistically efficient [19]. As a matter of fact, according to the invariance principle of maximum likelihood (ML) estimators, an estimator of a function of the model parameter estimates is asymptotically efficient if the model parameter estimate is statistically efficient. Translating these results to the specific case of controller tuning, arguments have been put forward in favor of model-based approaches [6]. In fact, based on the translation of the previous results to controller estimation, it can be argued that an efficient model-based approach is optimal and will therefore achieve equivalent or better results than data-driven approaches that are not statistically efficient.

Analysis of the accuracy of controller estimates is limited both for data-driven and model-based approaches and a quantitative comparison confirming the argument given above is lacking. One of the problems in performing such an analysis is that the achieved performance of model-based controller tuning methods strongly depends on the modeling technique that is used. If an identified parametric model is used, the control performance depends on the identification approach and the resulting amount of under-modeling. Furthermore, the order of the controller depends in general on the order of the identified model. In practice, bounds on the modeling error can be defined, but the exact amount of under-modeling will be unknown and problem dependent.

In this paper, a model-based controller tuning approach based on the invariance principle of ML estimators is proposed that allows for a comparison of the asymptotic variance of the controller parameter estimate with the accuracy achieved by data-driven approaches. A high-order model is identified using ML estimation (in this step the modeling error can be assumed negligible) and the controller parameters are estimated using an $L_{2}$ approach, under the assumption that the control objective is achievable. According to the arguments set out above, this approach achieves the CramérRao lower bound [6]. Moreover, this method can fairly be compared to non-iterative data-driven control (in this work, the Correlation-based Tuning, CbT [21], will be accounted for) as both approaches are based on convex optimization 


\section{CONFIDENTIAL. Limited circulation. For review only.}

only. However, from the perspective of control design, the variance analysis of the controller parameters is only an intermediate step towards the evaluation of the methods. In fact, the real final objective is the control cost achieved by the designed controller.

In this work, the accuracy of this final control objective is analyzed. By doing so, a more direct analysis of the performance will be carried out. The main conclusions of this paper are the following:

- if the model structure is perfectly known and the model order is low, the model-based approach is theoretically always the best in terms of statistical performance, as argued in [6];

- if the model structure is not completely known and/or a high-order model is identified using a ML estimator as indicated above, the data-driven approach can statistically outperform the model-based solution in terms of the control cost, even if the variance of the parameters remains larger.

- Since in the real world the model structure is never perfectly known and under-modeling cannot be avoided with a low-order model, the data-driven approach may give better results in real applications.

The remainder of the paper is as follows. Preliminaries and notation are given in Section II. The model-based and datadriven methods used in the paper for fixed-order modelreference design are described in Section III. The main results on accuracy analysis are presented in Section IV. A simulation example is used in Section $\mathrm{V}$ to illustrate the theoretical observations on the benchmark system introduced in [12]. Finally, Section VI concludes the paper.

\section{Preliminaries}

\section{A. The approximate model reference control problem}

Consider the stable linear SISO plant $G\left(q^{-1}\right)$, where $q^{-1}$ denotes the backward shift operator. Specifications for the controlled plant are given as a reference model $M\left(q^{-1}\right)$. In the following, it is assumed that $M \neq 1$. The backward shift operator will be omitted in the sequel for convenience. The control objective is to design the controller $K(\rho)$, parameterized through $\rho$, such that the closed-loop system resembles the reference model $M$. This can be achieved by minimizing the two-norm of the difference between the reference model and the achieved closed-loop system:

$$
J_{m r}(\rho)=\left\|M-\frac{K(\rho) G}{1+K(\rho) G}\right\|_{2}^{2}
$$

A discussion on the choice of $M$ can be found in [2]. In the following, the controller structure is chosen linear in the parameters,

$$
K\left(q^{-1}, \rho\right)=\beta^{T}\left(q^{-1}\right) \rho, \quad \rho \in \mathcal{D}_{K} \subseteq \mathbb{R}^{n_{\rho}}
$$

where the set $\mathcal{D}_{K}$ is compact and

$$
\beta\left(q^{-1}\right)=\left[\beta_{1}\left(q^{-1}\right), \cdots, \beta_{n_{\rho}}\left(q^{-1}\right)\right]^{T}
$$

is a vector of size $n_{\rho}$ of linear discrete-time transfer operators (in general an orthogonal basis). Only the cases where $K(\rho)$ is stable or it contains an integrator if $M(1)=1$ will be considered.

The ideal controller $K^{*}$ can be defined indirectly by $G$ and $M$ as

$$
K^{*}=\frac{M}{G(1-M)},
$$

that always exists since $M \neq 1$. Notice that $K^{*}$ might be of very high order, it might not stabilize the plant internally and it might be non-causal.

Notice that the model reference criterion (1) is non-convex with respect to $\rho$. An approximation that is convex for linearly parameterized controllers (2) can be defined using the reference model, as follows. The ideal sensitivity function is given by

$$
\frac{1}{1+K^{*} G}=1-M
$$

Note that this function is causal (as well as the reference model $M$ ) independent of the causality of $K^{*}$. Recalling (4), the model reference criterion (1) can be expressed as:

$$
J_{m r}(\rho)=\left\|\frac{K^{*} G-K(\rho) G}{\left(1+K^{*} G\right)(1+K(\rho) G)}\right\|_{2}^{2}
$$

Approximation of $1 /(1+G K(\rho))$ by $1-M$, the ideal sensitivity function, leads to the following approximation of the model reference criterion:

$$
\begin{gathered}
J(\rho)=\left\|\frac{K^{*} G-K(\rho) G}{\left(1+K^{*} G\right)^{2}}\right\|_{2}^{2}= \\
=\|(1-M)[M-K(\rho)(1-M) G]\|_{2}^{2} .
\end{gathered}
$$

The quality of this approximation of $J_{m r}(\rho)$ is discussed in [1]. Notice that, with the selected parameterization, $J(\rho)$ is a quadratic function of $\rho$ and its global optimizer can be easily found using the least squares techniques.

The optimal controller is defined as $K_{o}=K\left(\rho_{o}\right)$ with

$$
\rho_{o}=\arg \min _{\rho \in \mathcal{D}_{K}} J(\rho)
$$

In practice, if the controller order is fixed according to (2), the objective is not necessarily achievable and $K^{*} \notin$ $\{K(\rho)\}, K_{o} \neq K^{*}$ and $J\left(\rho_{o}\right)>0$. To allow for analysis of the accuracy of the estimated controller parameters, it is assumed that

A1 The objective can be achieved, i.e. $K^{*} \in\{K(\rho)\}$. Therefore, it holds that $K_{o}=K\left(\rho_{o}\right)=K^{*}$ and $J\left(\rho_{o}\right)=0$.

\section{B. System identification}

Assume that a set of input, $r(t)$, and output data, $y(t)$, with data length $N$ is available from an open-loop experiment. Suppose that the output is generated as:

$$
y(t)=G\left(q^{-1}\right) r(t)+v(t)
$$

where $v(t)$ is the measurement noise.

From the point of view of system identification, many different approaches can be employed to identify the system 
dynamics. In this paper, an FIR model $\hat{G}$ of $G$ will be identified, as the optimization is convex and does not require any prior knowledge on the system structure, except for the length of its impulse response (that however can be inferred from data, if the energy of noise is low).

Introduce the impulse response $g(t)$ of $G$ and $\theta_{o}=$ $[g(0) \ldots g(n-1)]^{T}$, where $n$ the length of the impulse response, such that $g(t) \approx 0, t \geq n$. Note now that (9) can be rewritten as $y(t) \approx \psi^{T}(t) \theta_{o}+v(t)$, where

$$
\psi(t)=[r(t) \ldots r(t-n+1)]^{T} .
$$

An FIR estimate of $G$ of length $n$ is given by:

$$
\hat{\theta}=\left[\frac{1}{N} \sum_{t=1}^{N} \psi(t) \psi^{T}(t)\right]^{-1} \frac{1}{N} \sum_{t=1}^{N} \psi(t) y(t) .
$$

Assume now that

A2 The measurement noise $v(t)$ is uncorrelated with $r(t)$.

A3 The measurement noise can be represented as $v(t)=H_{v} e(t)$, where $e(t)$ is a zero-mean white noise signal with variance $\sigma^{2}$ and bounded fourth moments. $H_{v}$ and $H_{v}^{-1}$ are stable filters.

A4 $\quad r(t)$ is persistently exciting of order $n$ and (1 $M)^{2} G$ has no zero on the imaginary axis.

A5 The FIR model order is such that $n \geq n_{\rho}$.

The estimate (10) provides a unique solution, if $\mathbf{A 4}$ is satisfied, given by $\hat{\theta}=\theta_{o}+\tilde{\theta}$, where

$$
\tilde{\theta}=\left[\frac{1}{N} \sum_{t=1}^{N} \psi(t) \psi^{T}(t)\right]^{-1} \frac{1}{N} \sum_{t=1}^{N} \psi(t) v(t) .
$$

This estimate is consistent: $\lim _{N \rightarrow \infty} \hat{\theta}=\theta_{o}$, w.p.1, [13] Moreover, if $v(t)$ is white, (10) is a maximum-likelihood (ML) estimator and the Cramér-Rao lower bound for the variance is achieved. When this is the case, the following principle (Theorem 5.1.1 [23]) holds, regarding all quantities derived from $\hat{\theta}$.

Invariance principle of maximum-likelihood estimation: Let $f: \Theta \rightarrow \Omega$ be a function mapping $\theta \in \Theta \in \mathbf{R}^{n}$ to an interval $\Omega \in \mathbf{R}^{m}$, with $m \leqslant n$. The invariance principle of ML estimation then states that, if $\hat{\theta}$ is a ML estimator of $\theta$, then $f(\hat{\theta})$ is a ML estimator of $f(\theta)$.

\section{MODEL REFERENCE CONTROL DESIGN FROM DATA}

\section{A. The correlation approach}

Consider the scheme in Fig. 1, when $v=0$ and the reference signal $r(t)=u(t)$, with $u(t)$ a white noise of unit variance. This scheme can be used to derive the optimal controller without using any explicit mathematical model of the process.

As a matter of fact, the most important observation at the basis of the $\mathrm{CbT}$ rationale is that, in the noiseless setting,

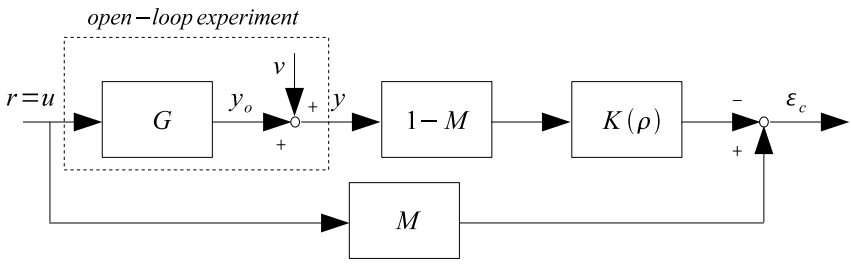

Fig. 1. Tuning scheme for Correlation-based Tuning

the error signal $\varepsilon_{c}(t, \rho)$ can be directly computed from I/O data as follows:

$$
\begin{gathered}
\varepsilon_{c}(t, \rho)=M r(t)-(1-M) K(\rho) G r(t) \\
=M u(t)-(1-M)) K(\rho) y(t)
\end{gathered}
$$

and, assuming $\mathbf{A 1}$ holds, the minimizer of the two-norm of $\varepsilon_{c}(t, \rho)$ is exactly $K_{o}$.

When data are collected in a noisy environment, the method resorts to the correlation approach to identify the controller. Specifically, an extended instrumental variable $\zeta(t)$ correlated with $u(t)$ and uncorrelated with $v(t)$ is introduced to decorrelate the error signal $\varepsilon_{c}(t)$ and $u(t) . \zeta(t)$ is defined as

$$
\zeta(t)=[u(t+l), \ldots, u(t), \ldots, u(t-l)]^{T},
$$

where $l$ is a sufficiently large integer. The correlation function is defined as

$$
f_{N, l}(\rho)=\frac{1}{N} \sum_{t=1}^{N} \zeta(t) \varepsilon_{c}(t, \rho)
$$

and the correlation criterion as

$$
J_{N, l}(\rho)=f_{N, l}^{T}(\rho) f_{N, l}(\rho) .
$$

In [21], it has been proven that

$$
\lim _{N, l \rightarrow \infty, l / N \rightarrow 0} J_{N, l}(\rho)=J(\rho),
$$

for any sufficiently exciting input sequence, if data in $\zeta(t)$ are prefiltered by $L_{c}\left(q^{-1}\right)$, defined as

$$
L_{c}\left(e^{-j \omega}\right)=\frac{1-M\left(e^{-j \omega}\right)}{\Phi_{u}(\omega)},
$$

where $\Phi_{u}(\omega)$ denotes the spectral density of $u(t)$. Notice that such a prefilter may be non-causal but it can be implemented off-line.

The optimal controller is then defined as $K_{C b T}=K\left(\hat{\rho}_{C b T}\right)$ with

$$
\hat{\rho}_{C b T}=\arg \min _{\rho \in \mathcal{D}_{K}} J_{N, l}(\rho)
$$

\section{B. Model-based model reference control}

If a model $\hat{G}$ of the system is available, a model reference controller $\hat{K}$ can be computed as

$$
\hat{K}=\frac{M}{\hat{G}(1-M)} \text {. }
$$

However, in any model-reference method this might lead to a high-order controller that may destabilize the system if $M$ is not minimum phase. $\mathcal{H}_{2}$ control theory can be used 
to compute a full-order model reference controller followed by a controller order reduction technique to compute a fixed-order controller. The accuracy of the final (fixed-order) controller is difficult to compute.

An alternative design of a fixed-order controller by minimization of the model reference criterion (1) approximated using the model $\hat{G}$ leads to a non-convex optimization approach. The quality of this controller estimate will depend on the initial values of the optimization variables and a fair comparison with data-driven approaches based on convex optimization is not possible. In this paper, the approximate control criterion (7) used in the data-driven approaches is therefore considered to develop a model-based approach that is comparable to the data-driven approaches.

Specifically, the approximate model reference criterion (7) can be approximated using the model $\hat{G}$ of the plant $G$, by minimizing $\|(1-M)[M-K(\rho)(1-M) \hat{G} \|$ over $\rho$. Since a parametric model is available, a simulated output sequence can be generated. This sequence can then be used to approximate the control criterion. This approach has also been used in model reduction, i.e. [19], [18].

In the following, a high-order parametric model $\hat{G}$ parametrized through $\hat{\theta}$ with an FIR structure is used together with the impulse excitation signal $\delta(t)$ to generate a simulated impulse response sequence, $y_{\hat{\theta}}(t)=\hat{G} \delta(t)$. This simulated output can be used to minimize the approximate model reference criterion

$$
\begin{gathered}
\hat{\rho}_{\hat{\theta}}=\arg \min _{\rho \in \mathcal{D}_{K}} J_{m b}(\rho, \hat{\theta}) \\
J_{m b}(\rho, \hat{\theta})=\frac{1}{N_{\delta}} \sum_{t=1}^{N_{\delta}}\left(s(t)-K(\rho)(1-M)^{2} y_{\hat{\theta}}(t)\right)^{2}
\end{gathered}
$$

where $s(t)$ is the impulse response of $(1-M) M$, i.e. $s(t)=$ $(1-M) M \delta(t)$ and the number of generated samples $N_{\delta} \geq n$. The error can be written as:

$$
s(t)-K(\rho)(1-M)^{2} y_{\hat{\theta}}(t)=s(t)-\phi_{\hat{\theta}}^{T}(t) \rho,
$$

where the regression vector $\phi_{\hat{\theta}}(t)$ is given by

$$
\begin{gathered}
\phi_{\hat{\theta}}(t)=\beta(1-M)^{2} y_{\hat{\theta}}(t)= \\
=\beta(1-M)^{2} G \delta(t)+\beta(1-M)^{2} \Delta G \delta(t) \triangleq \phi_{o}(t)+\tilde{\phi}_{\hat{\theta}}(t),
\end{gathered}
$$

and $\Delta G=\hat{G}-G$. The minimizer of (19) is given by

$$
\hat{\rho}_{\hat{\theta}}=\left[\frac{1}{N_{\delta}} \sum_{t=1}^{N_{\delta}} \phi_{\hat{\theta}}(t) \phi_{\hat{\theta}}^{T}(t)\right]^{-1} \frac{1}{N_{\delta}} \sum_{t=1}^{N_{\delta}} \phi_{\hat{\theta}}(t) s(t)
$$

For simplicity, from now on, let $N_{\delta}=N$ without loss of generality.

Proposition 1: Assume that A1, A2, A3, A4, A5 are satisfied and let $N>n$. Then, if the FIR model $\hat{\theta}$ is estimated according to (10) and the controller parameters $\hat{\rho}_{\theta}$ according to $(23)$,

$$
\lim _{N \rightarrow \infty} \hat{\rho}_{\hat{\theta}}=\rho_{o}, \text { w.p.1. }
$$

Proof: The noise-free signal $s(t)$ can be written as $s(t)=\phi_{\hat{\theta}}^{T}(t) \rho_{o}-\tilde{\phi}_{\hat{\theta}}(t) \rho_{o}$, the estimation error is given by

$$
\hat{\rho}_{\hat{\theta}}-\rho_{o}=-\left[\frac{1}{N} \sum_{t=1}^{N} \phi_{\hat{\theta}}(t) \phi_{\hat{\theta}}^{T}(t)\right]^{-1} \frac{1}{N} \sum_{t=1}^{N} \phi_{\hat{\theta}}(t) \tilde{\phi}_{\hat{\theta}}^{T}(t) \rho_{o} .
$$

Since $\lim _{N \rightarrow \infty} \hat{\theta}=\theta_{o}$, a continuous function of this variable $f(\hat{\theta})$ converges w.p. 1 to $f\left(\theta_{0}\right)$ ([14], page 450). Consequently $\lim _{N \rightarrow \infty} \tilde{\phi}_{\hat{\theta}}(t)=0$, w.p. 1 , the regressor converges to the noise-free regressor, $\lim _{N \rightarrow \infty} \phi_{\hat{\theta}}(t)=\phi_{o}(t)$, w.p.1, and

$$
\lim _{N \rightarrow \infty} \frac{1}{N} \sum_{t=1}^{N} \phi_{\hat{\theta}}(t) \phi_{\hat{\theta}}^{T}(t)=R_{o}, \quad \text { w.p.1, }
$$

with $R_{O}$ defined as

$$
R_{o}=\lim _{N \rightarrow \infty} \frac{1}{N} \sum_{t=1}^{N} \phi_{o}(t) \phi_{o}^{T}(t) .
$$

This matrix has full rank since $N \geq n$ and $\mathbf{A 4 , ~ A 5 ~ h o l d . ~ I t ~}$ follows that $\lim _{N \rightarrow \infty}\left(\hat{\rho}_{\hat{\theta}}-\rho_{o}\right)=0$, w.p.1, which completes the proof.

\section{ACCURACY ANALYSIS}

\section{A. Variance analysis}

For the correlation approach, if $\mathbf{A 1}$ holds, the error between the estimated controller parameters $\hat{\rho}_{C b T}$ and the optimal controller parameters $\rho_{o}$ is asymptotically normally distributed and the asymptotic covariance matrix of $\sqrt{N}\left(\hat{\rho}_{C b T}-\right.$ $\left.\rho_{o}\right)$ is given by [17]:

$$
P_{c}=\sigma^{2}\left(Q^{T} Q\right)^{-1} Q^{T} S Q\left(Q^{T} Q\right)^{-1}
$$

where

$$
\begin{aligned}
Q & =\lim _{N \rightarrow \infty} \frac{1}{N} \sum_{t=1}^{N} \zeta(t) \phi_{o}^{T}(t) \\
S & =\lim _{N \rightarrow \infty} \frac{1}{N} \sum_{t=1}^{N}\left[H^{*} \zeta(t)\right]\left[H^{*} \zeta(t)\right]^{T} .
\end{aligned}
$$

and $H^{*}=K^{*}(1-M) H$.

For model-based control, the accuracy of the estimate $\hat{\rho}_{\hat{\theta}}$ of (23) clearly depends on the accuracy of the estimate of the model parameters $\hat{\theta}$ defined in (10). However, the invariance principle of ML estimation provides a condition on $\hat{\theta}$ that assures that $\hat{\rho}_{\theta}$ is statistically efficient. As a matter of fact, according to the invariance principle, $\hat{\rho}_{\theta}$ is a ML estimator of $\rho_{o}$ if $\hat{\theta}$ is a ML estimator of $\theta_{o}$. If the measurement noise is white (i.e., $H=1$ ), the FIR estimate $\hat{\theta}$ is a ML estimator, whose variance corresponds to the Cramér-Rao bound, and also $\hat{\rho}_{\theta}$ is a ML estimate. Specifically, the Cramér-Rao bound for the function $f(\theta)$ of the ML estimate $\theta$ is given by

$$
\frac{\partial f(\theta)}{\partial \theta} P_{\theta} \frac{\partial f(\theta)}{\partial \theta}
$$

where $P_{\theta}$ is the Cramér-Rao bound for the estimate $\hat{\theta}$ [10]. The best variance that can be achieved thus depends on the 
function $f(\theta)$. Results from asymptotic analysis in system identification can be used to calculate the Cramér-Rao bound for $f(\theta)$ as illustrated by the following Proposition.

Proposition 2: Assume that $N>n$. Then, if $\hat{\theta}$ is estimated according to (10) and $\hat{\rho}_{\hat{\theta}}$ according to (23), $\sqrt{N}\left(\hat{\rho}_{\hat{\theta}}-\right.$ $\left.\rho_{o}\right)$ is asymptotically normally distributed with covariance matrix $P_{m b}$ :

$$
P_{m b}=\sigma^{2} R_{o}^{-1} C R_{o}^{-1},
$$

where $C$ is defined as

$$
C=\lim _{N \rightarrow \infty} \frac{1}{N} \sum_{t=1}^{N}\left[H^{*} \phi_{o}(t)\right]\left[H^{*} \phi_{o}(t)\right]^{T}
$$

Proof: The proof is based on Theorem 9.1 of [13]. It can be shown that the estimate $\hat{\rho}_{\theta}$ satisfies the assumptions of Theorem 9.1 of [13]. A complete proof can be found in [20].

According to the previous analysis, the given model-based control design method using any full-order model is statistically efficient if the noise is white, whereas the proposed data-driven technique is not, for any $H$. In the following, it will be shown that this does not imply that the model based approach achieves better control performance.

\section{B. The control objective}

The main idea behind data-driven methods is that the model of the system to control is only an intermediate step towards the final controller tuning phase, and therefore it might be better to directly focus on the final objective, to avoid the risk of losing some information in undermodeling. According to this mindset, also the variance of the parameters is only an intermediate step towards the evaluation of what happens to the control criterion when data are noisy and $N$ is large, but finite.

In this subsection, the effect of noise will be assessed on the capability of the control design criteria of estimating (7). The estimate will be shown to be biased when $N$ is large but finite and therefore the average model-matching error will be greater than zero even when $\mathbf{A 1}$ holds. It will be also shown that, from this point of view, a criterion based on a ML estimator of the model is not always statistically better, in terms of the control cost (7), than data-driven design.

Concerning $\mathrm{CbT}$, the following result holds, already proven in [21].

Proposition 3: For large $N$, the expected value of the correlation criterion (14) is as follows.

$$
\mathbb{E}\left[J_{N, l}(\rho)\right] \approx J(\rho)+\frac{\sigma^{2}(2 l+1)}{2 \pi N} \int_{-\pi}^{\pi} \frac{|1-M|^{4}|K(\rho)|^{2}|H|^{2}}{\Phi_{u}(\omega)} d \omega .
$$

Proof: See [21].

The same approach applied to model-reference control using model-based formula (23) gives the following bias for the control cost for large and finite $N$.

Proposition 4: For large $N$, the expected value of the model-based cost function (23) is as follows.

$$
\mathbb{E}\left[J_{m b}(\rho)\right] \approx J(\rho)+\frac{\sigma^{2} n}{2 \pi N} \int_{-\pi}^{\pi} \frac{|1-M|^{4}|K(\rho)|^{2}|H|^{2}}{\Phi_{u}(\omega)} d \omega .
$$

Proof: Consider again the model-based control cost (19) and define $\Delta G=G-\hat{G}$. Since $\hat{y}_{\theta}(t)=\hat{G} \delta(t)$, where $\delta(t)$ is the discrete-time impulse, the control cost is given by

$$
\begin{gathered}
J_{m b}(\rho)=\frac{1}{N} \sum_{t=1}^{N}\left(s(t)-K(\rho)(1-M)^{2} \hat{y}_{\theta}(t)\right)^{2}= \\
=\frac{1}{N} \sum_{t=1}^{N}\left(s(t)-K(\rho)(1-M)^{2} G \delta(t)\right)^{2}+ \\
+\frac{1}{N} \sum_{t=1}^{N}\left(K(\rho)(1-M)^{2} \Delta G \delta(t)\right)^{2}+ \\
+\frac{2}{N} \sum_{t=1}^{N}\left(s(t)-K(\rho)(1-M)^{2} G \delta(t)\right)\left(K(\rho)(1-M)^{2} \Delta G \delta(t)\right) .
\end{gathered}
$$

Notice that the first term of the sum is a (noiseless) consistent estimator of $J(\rho)$. Since the estimate of $G$ is consistent, i.e. $\mathbb{E}[\Delta G]=0$, then the expectation of $J_{m b}(\rho)$ becomes

$$
\mathbb{E}\left[J_{m b}(\rho)\right]=J(\rho)+\frac{1}{N} \sum_{t=1}^{N} \mathbb{E}\left[\left(K(\rho)(1-M)^{2} \Delta G \delta(t)\right)^{2}\right]
$$

and its Parseval counterpart is

$$
\mathbb{E}\left[J_{m b}(\rho)\right]=J(\rho)+\frac{1}{2 \pi} \int_{-\pi}^{\pi}|1-M|^{4}|K(\rho)|^{2} \mathbb{E}\left[|\Delta G|^{2}\right] \Phi_{\delta}(\omega) d \omega .
$$

In the literature [13], it is well-known that for high order models the following approximation holds

$$
\mathbb{E}\left[|\Delta G|^{2}\right] \approx \frac{n}{N}|H|^{2} \sigma^{2} \Phi_{u}^{-1}(\omega)
$$

Moreover, being $\delta$ an impulse, $\Phi_{\delta}(\omega)=1, \forall \omega$ and therefore (31) holds, which completes the proof.

Propositions 3 and 4 indicate that:

- both the data-driven and the model-based criteria $J_{N, l}$ and $J_{m b}$ are biased and the bias depends on $\rho$;

- the bias is composed by an integral term (equal in (31) and (30)) and a coefficient that is different in the two cases;

- depending on $l / n$, the bias will be larger in one case or in the other.

The results of the previous analysis will be commented upon in detail in the next subsection.

\section{Discussion}

The results of the last subsection are interesting as they evaluate the average behaviour of the model-based and data-driven controllers from a different view than standard statistical analysis. This new perspective highlights some critical points that should be evaluated before drawing final conclusions about the comparison of model-based and datadriven approaches.

In standard statistical analysis, the performance of an estimator that is asymptotically consistent is evaluated by means of the asymptotic variance. The method that achieves the lowest asymptotic variance is usually considered to be the best estimator. If such an evaluation, combined with 
the invariance principle of maximum-likelihood estimation, is applied to the controller design methods discussed in this paper, the model-based design approach (that achieves optimal asymptotic variance) can be considered the best estimator. However, the results in Propositions 3 and 4 show that the expectation of the final control criterion is lower in the data-driven case, when the model is high-order and $n>2 l+1$. The reason for this discrepancy is that the analysis based on asymptotic variance does not take into account the other factors affecting the final control criterion, i.e. $l$ and $n$. These design parameters offer a trade-off between the minimizer of the real criterion to minimize, i.e. J, and the minimizer of a bias term that is null if $\rho=0$. Notice that the case of $n>2 l+1$ is all but unlikely in real-world applications. As a matter of fact, $l$ should be close to the length of the impulse response of $M-K G(1-M)$, which is unknown. However, standing on the assumption that it is possible to match most of $M$ with $K$, the choice of $l$ equal to the length of the impulse response of $M$ is sufficient. For the condition $n>2 l+1$ to be satisfied, it is then sufficient that the settling time of the FIR model $\hat{G}$ is larger than that of $M$ or $\hat{G}$ is low-damped (see Section V).

In standard practice of model-based design, when the system is complex and a low order model is not sufficient to accurately describe the I/O dynamics, one may think that increasing the order is the best way to find a good model. For what said above, one of the main conclusions of this paper is that this is not generally true if the model has to be used for control design. A data-driven method, that does not depend on a model of the system, might be a better solution instead.

Furthermore, it should also be considered that the "order" of a real system is a badly defined concept. Every model is only an approximation of the real world. It follows that the data-driven method might outperform the model-based method also when the model is low-order. In the following section, it will be shown that this might happen even when the model error is very small and when standard procedures for system identification are followed.

Finally, notice that the data-driven approach is convex if the controller is linearly parameterized, whereas in the modelbased approach, both the model and the controller need to be linearly parameterized to obtain convexity. These observations make data-driven techniques appealing for the practical use.

\section{NuMERICAL EXAMPLE}

\section{A. The benchmark system}

The flexible transmission system proposed as a benchmark in [12] was used in [1], [16] and [9] to illustrate data-driven controller tuning approaches. The same example is used here. The plant is given by the discrete-time model

$$
G\left(q^{-1}\right)=\frac{0.28 q^{-3}+0.51 q^{-4}}{1-1.42 q^{-1}+1.59 q^{-2}-1.32 q^{-3}+0.89 q^{-4}} .
$$

The controller structure is given as

$$
K(\rho)=\frac{\rho_{1}+\rho_{2} q^{-1}+\rho_{3} q^{-2}+\rho_{4} q^{-3}+\rho_{5} q^{-4}+\rho_{6} q^{-5}}{1-q^{-1}} .
$$

PRBS signals with unity amplitude are used as input to the system, $r(t)$. The output of the plant is disturbed by zeromean white noise $v(t)$. Results are given for $N=1000$, sampling time $T_{s}=50 \mathrm{~ms}$ and increasing length of the instrumental variable $l$. A Monte-Carlo simulation with 100 experiments is performed, using a different noise realization for each experiment, for a signal-to-noise ratio (SNR) of 10 in terms of standard deviation. The noise realizations are the same for all methods. The reference model is defined as

$$
M\left(q^{-1}\right)=\frac{K\left(\rho_{o}\right) G}{1+K\left(\rho_{o}\right) G}
$$

with

$$
\rho_{o}=[0.2045,-0.2715,0.2931,-0.2396,0.1643,0.0084]^{T} .
$$

The optimal controller $K\left(\rho_{o}\right) \in\{K(\rho)\}$ and the objective can be achieved. In Figure (2), the impulse response of $G$ and $M$ are illustrated. Since the number of nonzero samples is (almost) 180 for $G$ and (almost) 35 for $M$, an FIR model with $n=180$ is used in the model-based approach whereas for $\mathrm{CbT} l=35$ is selected.

The results of the 100 Monte Carlo runs for the model-based

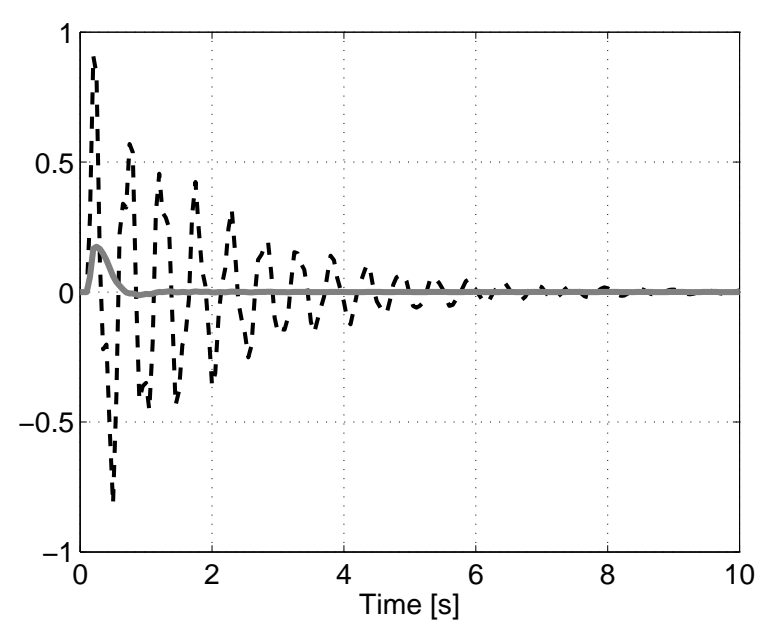

Fig. 2. Impulse response of $G$ (dashed) and $M$ (solid).

design using an FIR model with $n=180$, for CbT with $l=35$ and for CbT with $l=130$ are summarized in Table I. Two estimates of $\mathbb{E}[J(\rho)]$ and $\mathbb{E}\left[J_{m r}(\rho)\right]$ are calculated, respectively, as

$$
V_{c}=\frac{1}{100} \sum_{i=1}^{100} J\left(\hat{\rho}^{(i)}\right)
$$

and

$$
V_{m r}=\frac{1}{100} \sum_{i=1}^{100} J_{m r}\left(\hat{\rho}^{(i)}\right)
$$


where $\hat{\rho}^{(i)}$ is the controller parameter vector at the $i^{t h}$ Montecarlo run, and the average trace of the parameter variance

$$
V_{t}=\frac{1}{100} \sum_{i=1}^{100} \operatorname{tr}\left\{\operatorname{var}\left[\hat{\rho}^{(i)}\right]\right\}
$$

is also given. For comparison, the performance achieved using low order models estimated using the OE approach is finally presented.

As predicted by the theory of Propositions 3 and 4, the average of the cost criterion is lower in the data-driven case when $n>2 l+1$, even if the parameter variance is larger. When $l$ is overestimated, e.g. when $l=130$ and $n<2 l+1$, the variance of the model-based design remains smaller, but now the average of the control cost is also lower than that for the data-driven design.

If both the model structure (OE) and the model order are known, the low order model-based solution outperforms the data-driven approach (note that in this case, since the order of the real system $n=4$ is low, the result of Proposition 4 no longer holds). However, this does not mean that the modelbased approach is more suitable in practice. In the real-world, a "full-order model" does not exist and any description is by definition an approximation. The results presented in Table I show that even a small under-modeling error may jeopardize the control performance. The case where an OE model with the right number of poles and the right relative degree but without zeros introduces a modeling error that is very likely in practice. As a matter of fact, note that the physics usually suggest the order of the model but not the exact number of zeros, especially in discrete time. The identified model is very similar to the real system, as illustrated in Figure 3 and the user may believe that this is an accurate description of the system, but the resulting controller does not yield good control performance. The same observations can be made for the case where the relative degree is 4 instead of 3 (only one more than the "real" system).

The average of the achieved original model-reference criterion $J_{m r}$ is reported to show that the approximate criterion $J$ is a good approximation and that therefore the conclusions hold for the original model reference criterion, even if the analysis has been carried out with respect to the convexified one. The results show that $J_{m r}$ and $J$ are very similar for the FIR and CbT approaches as well as the low-order model approach when no under-modeling is present. In the case of under-modeling in the model-based approach, the approximation is less good since (19) depends on the model (and not on system) dynamics. As a result, the model reference control cost (1) is larger than (7), which further encourages the use of a data-driven technique.

This example then shows that:

- standard, statistically efficient model-based approaches achieve better performance than the data-driven solution considered in this paper only if the correct model structure and order are used;

- the data-driven approach can outperform a statistically efficient model-based solution based on a high-order

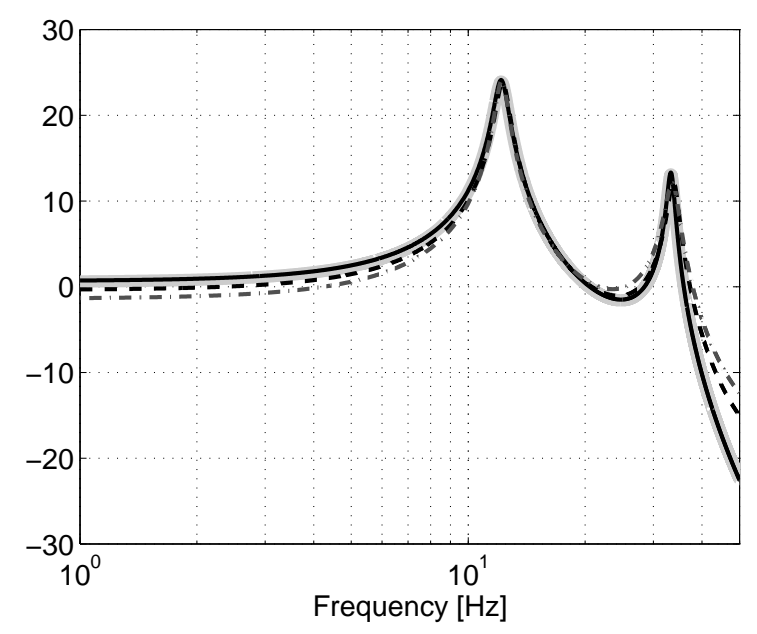

Fig. 3. Output error modeling of $G$ : magnitude of the frequency response of the real plant (thick grey line), of the $\mathrm{OE}(2,4,3)$ model (solid black line), of the $\mathrm{OE}(1,4,3)$ model (dashed black line) and of the $\mathrm{OE}(2,4,4)$ model (dash-dotted grey line).

model (if $n>2 l+1$ );

- the data-driven approach can outperform a statistically efficient model-based solution in case of (slight) undermodeling.

\section{CONCLUSIONS}

In this paper, the accuracy of data-driven non-iterative controller tuning is compared to the accuracy of a modelbased approach using a maximum likelihood estimator, in the case where the control objective can be achieved. Data-driven non-iterative controller tuning approaches lead to a non standard identification problem, where estimates are consistent also if the control objective cannot be achieved, but they are statistically not optimal [22], i.e. the CramérRao bound is not reached. It could therefore be argued that, from a statistical point of view, it is always better to first identify a model and then design a model-based controller. However, this assessment of the statistical properties does not look at the final control objective.

In this paper, it has been shown that the expected value of the final control cost is biased and the bias depends not only on the variance of the controller parameters, but also on some parameters. Specifically, in CbT, the bias is affected by the length of the instrumental variable, while in the model-based approach it is influenced by the model order. It might therefore happen that for large but finite number of data, a data-driven approach achieves a lower control cost than a statistically efficient model-based approach, as illustrated in the proposed numerical example. In the paper, it is also shown that, when applied to real systems, also the best model found via standard identification techniques can be outperformed by a data-driven method. This is due to the fact that in a real setup, a "full-order" model does not exists and every description is by definition an approximation of the reality. 


\section{CONFIDENTIAL. Limited circulation. For review only.}

TABLE I

ACHIEVED PERFORMANCE (35), (36) AND (37) OVER 100 RUNS FOR MODEL-BASED (MB) AND DATA-DRIVEN (CBT) DESIGN.

\begin{tabular}{|c|c|c|c|c|c|c|}
\hline & \multicolumn{4}{|c|}{ MB } & \multicolumn{2}{|c|}{$\mathrm{CbT}$} \\
\hline & $\mathrm{OE}(2,4,3)$ & $\mathrm{OE}(2,4,4)$ & $\mathrm{OE}(1,4,3)$ & FIR: $n=180$ & $l=35$ & $l=130$ \\
\hline$V_{t}\left(\times 10^{-3}\right)$ & 0.3676 & 0.3896 & 0.0144 & 0.7378 & 3.0578 & 0.9646 \\
\hline$V_{c}$ & 0.0064 & 0.0688 & 0.1332 & 0.0573 & 0.0375 & 0.0586 \\
\hline$V_{m}$ & 0.0064 & 0.0759 & 0.1424 & 0.0575 & 0.0376 & 0.0587 \\
\hline
\end{tabular}

The comparison in this paper is clearly limited, as, in the analysis, it is assumed that the control objective can be achieved. This will not be the case in practice, and the performance of different methods will be strongly case dependent. The results of this paper do show that the conclusion from [19] that "it is never better to estimate the (low order) model directly from data, compared to estimating it via $L_{2}$ model reduction of a high order FIR model" is true for reducedorder system identification but does not hold for controller tuning.

\section{REFERENCES}

[1] M. C. Campi, A. Lecchini, and S. M. Savaresi. Virtual reference feedback tuning: A direct method for the design of feedback controllers. Automatica, 38:1337-1346, 2002.

[2] A. Dehghani, A. Lanzon, and B. D.O. Anderson. $\mathrm{H}_{\infty}$ design to generalize internal model control. Automatica, 42(11):1959 - 1968, 2006.

[3] S. Formentin, A. Cologni, D. Belloli, F. Previdi, and S.M. Savaresi. Fast tuning of cascade control systems. In IFAC World Congress, volume 18, pages 10243-10248, 2011.

[4] S. Formentin, P. De Filippi, M. Corno, M. Tanelli, and S.M. Savaresi. Data-driven design of braking control systems. IEEE Transactions on Control Systems Technology, (to appear. DOI: 10.1109/TCST.2011.2171965).

[5] G. O. Guardabassi and S. M. Savaresi. Virtual reference direct design method: an off-line approach to data-based control system design. IEEE Transactions on Automatic Control, 45(5):954-959, 2000.

[6] H. Hjalmarsson. From experiment design to closed-loop control. Automatica, 41(3):393-438, 2005.

[7] H. Hjalmarsson, S. Gunnarsson, and M. Gevers. A convergent iterative restricted complexity control design scheme. In 33rd IEEE Conference on Decision and Control, volume 2, pages 1735-1740, December 1994.

[8] A. Karimi, L. Mišković, and D. Bonvin. Iterative correlation-based controller tuning. International Journal of Adaptive Control and Signal Processing, 18(8):645-664, 2004.

[9] A. Karimi, K. van Heusden, and D. Bonvin. Non-iterative data-driven controller tuning using the correlation approach. In European Control Conference, pages 5189-5195, Kos, Greece, 2007.

[10] S. M. Kay. Fundamentals of Statistical Signal Processing: Estimation Theory. Prentice-Hall, New Jersey, 1993.

[11] R. L. Kosut. Uncertainty model unfalsification for robust adaptive control. Annual Reviews in Control, 25:65-76, 2001.

[12] I. D. Landau, D. Rey, A. Karimi, A. Voda, and A. Franco. A flexible transmission system as a benchmark for robust digital control. European Journal of Control, 1(2):77-96, 1995.

[13] L. Ljung. System Identification - Theory for the User. Prentice Hall, NJ, USA, second edition, 1999.

[14] R. Pintelon and J. Schoukens. System Identification: A Frequency Domain Approach. IEEE Press, New York, USA, 2001.

[15] M. G. Safonov and T. C. Tsao. The unfalsified control concept and learning. IEEE Transactions on Automatic Control, 42(6), 1997.

[16] A. Sala and A. Esparza. Extensions to "virtual reference feedback tuning: A direct method for the design of feedback controllers". Automatica, 41(8):1473-1476, 2005.

[17] T. Söderström and P. Stoica. System Identification. Prentice-Hall, U.K., 1989.

[18] F. Tjärnström. Variance analysis of L2 model reduction when undermodeling - the output error case. Automatica, 39(10):1809-1815, 2003.
[19] F. Tjärnström and L. Ljung. L2 model reduction and variance reduction. Automatica, 38(9):1517-1530, 2002.

[20] K. van Heusden. Non-Iterative Data-Driven Model Reference Control. $\mathrm{PhD}$ thesis, Ecole Polytechnique Fédérale de Lausanne, Laboratoire d'Automatique, 2010.

[21] K. van Heusden, A. Karimi, and D. Bonvin. Data-driven model reference control with asymptotically guaranteed stability. International Journal of Adaptive Control and Signal Processing, 25(4):331-351, 2011.

[22] K. van Heusden, A. Karimi, and T. Söderström. On identification methods for direct data-driven controller tuning. International Journal of Adaptive Control and Signal Processing, 25(5):448-465, 2011.

[23] S. Zacks. The Theory of Statistical Inference. John Wiley, New York, 1979. 